"be directive and deviate from the tradition of nondirectiveness." Physicians were advised to recognize bias in their own opinions about abortion, Down syndrome, and so on, and if such bias existed, to refer to a genetics counselor for pretest counseling.

Now, I suppose it is possible that, somehow, research has shown a tendency toward improved maternal and fetal health among pregnant women who are not subjected to bias for or against abortion, Down syndrome, or other related subjects, when counseled regarding prenatal testing. It is possible but clearly very unlikely, and in any event, no such claim was make by the authors. It is not illegal to express such bias (yet). One is therefore left with the assumption that somehow the authors consider it inappropriate, unethical, or immoral to "be directive and deviate from the tradition of nondirectivenesss" when counseling regarding prenatal testing.

Several questions immediately arise. Whose tradition would be violated? The genetics counselors'? Is this tradition supported by any research into outcomes? Is it supported by any ethical reasoning? Let's be clear: the authors are recommending that, under certain circumstances, a referral is warranted. The referral is not being recommended on the grounds of lack of clinical competency or technical skills, or because a certain specialty is likely to have better clinical outcomes when handling a particular problem. So why is a referral being recommended? Why is being directive bad during genetics counseling when it is routine in other clinical circumstances? No physician thinks twice about being directive when recommending an appendectomy or a coronary artery bypass surgery. Why is counseling concerning prenatal testing any different? Another question concerns directiveness itself. Is there something wrong with being directive? If so, why are the authors being directive by directing us not to be directive? I don't want to be disingenuous. I strongly suspect the real point the authors are trying to make is that it is somehow wrong or unethical for a physician to recommend for or against prenatal testing or for or against an abortion based on the results of such testing. Such a concern might, I suppose, be based on an abortion rights basis or perhaps on a more general patient autonomy principle.

The abortion rights' position, while popular among certain segments of the population (including some physicians), does have certain philosophical problems. For instance, either it must deny the personhood of the unborn baby, or it must make that unborn baby's right to life subordinate to the mother's right to happiness. Other objections could be voiced as well. One must at the very least conclude that the issue is contentious; therefore, being directive based on such a position in a general clinical publication, without acknowledging one's own bias on the issue, is inappropriate. Because not all physicians share this particular bias, it would be more appropriate to acknowledge this bias is why a particular recommendation is being made rather than dogmatically assert (in this particular instance) that referral should be made.
The patient autonomy principle, while more widely acknowledged, is also philosophically questionable. Even if one accepts it at face value, however, many clinicians will acknowledge that there are two persons involved in a pregnancy, not one. My personal autonomy does not give me the right to do anything I want with my own body if, in so doing, I injure someone else. If the unborn is a human being, then it is immoral to kill him, since that would violate his personal autonomy. One can take the position that the unborn is not human. This position is illogical. Even those who reject the logic supporting the personhood of the unborn, however, must at least acknowledge the contentiousness of this issue. It is, again, inappropriate to make clinical recommendations that are based on a bias concerning such a contentious issue without acknowledging that bias.

Jeremy Klein, $M D$ Louisa, Ky

The above letter was referred to the authors of the article in question, who offer the following reply.

To the Editor: Nondirectiveness does have a long tradition for genetic service providers (both genetic counselors and clinical geneticists) in the Untied States. Nondirectiveness requires sharing all the relevant facts with the patient, but not telling the patient what to do. The issue is not that directiveness per se is bad, but that it is inappropriate in certain situations, including most genetic and prenatal diagnosis situations. Usually in prenatal diagnosis, the physician's knowledge, experience, and wisdom are not the most important factors in deciding a course of action; rather, it is the patient's beliefs and values. Thus, if a provider cannot assist the patient in recognizing his or her core beliefs and determining what course of action is most comfortable for that patient, the provider should refer to someone else who can provide that service.

Sarah Cate, MD, MPH Susie Ball, MS Central Washington Family Medicine Yakima

\section{Delivery of Preventive Services}

To the Editor: I would like to comment on the interesting study of predictors of screening for breast, cervical, colorectal, and prostatic cancer by Ruffin and colleagues. ${ }^{1}$ In an audit of community-based primary care practices, they made three key observations and one key interpretation. They observed that screening rates were suboptimal, that the strongest predictor of screening was scheduling a health maintenance visit, and that less than one third of patients have annual health maintenance examinations. These observations are in agreement with another community-based primary care audit of about 75,000 adults. ${ }^{2}$ Ruffin et al interpreted their findings to suggest that "the promotion of an annual visit to a health care provider to focus [on] preventive services is likely to increase the screening recommendations provided to patients and subsequent delivery of preventive services." 